\title{
结缔组织生长因子诱导肾成纤维细胞 转为成肌纤维细胞
}

\author{
黄海长 李惊子 王海燕
}

(北京大学第一医院肾内科, 北京大学肾脏病研究所卫生部重点实验室, 北京 100034. E-mail: haichang@ mail.bjmu.edu.cn)

\begin{abstract}
摘要 结缔组织生长因子(connective tissue growth factor, CTGF) 是介导转化生长因子(TGF- $\beta$ )促纤维化 效应的蛋白, 但它能否诱导肾脏慢性纤维化中的关键细胞, 即间质成纤维细胞转化为成肌纤维细胞 (myofibroblast), 目前尚无报道. 实验应用体外基因转染技术建立稳定表达 CTGF 蛋白的肾间质成纤维 细胞系, 并观察在不同培养条件下成肌纤维细胞数量及其产生的生物学效应. 结果发现细胞表达出的 CTGF 蛋白能直接诱导成纤维细胞转化为成肌纤维细胞, 并增加细胞外基质胶原 III mRNA水平, 提示 CTGF 可作为肾纤维化防治中的新靶位蛋白, 为临床延缓肾脏疾病慢性进展提供新思路.
\end{abstract}

\section{关键词 结缔组织生长因子 成纤维细胞 成肌纤维细胞 纤维化}

在慢性疾病状态或生理老化时, 多种组织脏器 如皮肤、肺、肝、肾、心的纤维化严重影响人类健 康. 肾脏纤维化是各种肾脏病慢性进展而最后导致 慢性肾功能衰竭的共同机制，而以肾间质细胞外基 质沉积为主要表现的肾间质纤维化过程是影响肾脏 功能的重要因素 ${ }^{[1]}$. 关于肾间质细胞外基质的来源, 目前认为是肾小管细胞、间质成纤维细胞以及浸润 的巨噬细胞分泌的, 但在病理状态下肾间质内存在 的另一种细胞一一成肌纤维细胞 (myofibroblasts) 是 间质细胞外基质持续增加的主要来源细胞 ${ }^{[2]}$. Ito 等 人 $^{[3]}$ 观察到, 肾间质纤维化病变区内结缔组织生长 因子(CTGF) mRNA 表达和成肌纤维细胞同步增加, 推测 CTGF 是参与肾间质纤维化的重要因子, 但 CTGF 和成肌纤维细胞形成是否有关联还不清楚. 本研究 通过基因转染、Western blot 蛋白分析、逆转录-PCR 等技术, 研究 CTGF 能否诱导肾间质成纤维细胞转 化为成肌纤维细胞, 以及对间质胶原 III mRNA 水平 的影响, 以进一步探讨 CTGF 在肾间质纤维化中的 作用机制.

\section{1 材料与方法}

(i) 主要试剂. 正常大鼠肾间质成纤维细胞 系(NRK-49F)来自美国 ATCC 公司, pRc-CMV-CTGF 基因真核稳定表达质粒由 School of Medicine, University of Miami 的 Grotendorst 教授惠赠, TGF$\beta 1$ 购自美国 R\&D System 公司, 抗 $\alpha$-平滑肌肌动蛋 白单克隆抗体购自美国 Sigma公司。 （ii）基因转染. 采用 Lipofectin 基因转移方法. NRK-49F 细胞在 5\% FBS DMEM 培养液中生长; 基 因转移具体步骤参照 LipofectAmine Plus ${ }^{\mathrm{TM}}$ 试剂 (GibcoBRL 公司)说明书.

(iii) 阳性细胞克隆的篎选、扩增和鉴定. $C T G F$ 基因经 Lipofectin 转染细胞后, 细胞在含 800 $\mu \mathrm{g} / \mathrm{mL} \mathrm{G} 418$ 的 5\% FBS DMEM 培养液笁选 3 4 周, 绝大部分细胞死亡, 再经 $1 \sim 2$ 周笁选培养, 可以看 见细胞克隆形成. 挑出细胞克隆, 经 96 孔 $\rightarrow 24$ 孔 $\rightarrow 6$ 孔扩增培养, 最后得到 $C T G F$ 基因稳定表达细胞系, 并维持 $200 \mu \mathrm{g} / \mathrm{mL} \mathrm{G} 418$ 培养液传代培养和实验研究. 裂解 $C T G F$ 基因表达细胞, 以抗-CTGF 抗体经 Western blot 蛋白分析方法鉴定 $C T G F$ 基因是否成功 转人细胞并表达 CTGF 蛋白; 也检测转染空载体质 粒(pRc-CMV)的细胞和正常 NRK-49F 细胞.

(iv) 细胞免疫组织化学染色. 细胞转种生长 于盖玻片上，收集细胞并以甲醇于 $-20^{\circ} \mathrm{C}$ 固定 $10 \mathrm{~min}$, 然后以常规 $\mathrm{ABC}$ 法进行成肌纤维细胞标记 $\alpha$-平滑肌 肌动蛋白 $(\alpha-S M A)$ 免疫组织化学染色. 以 PBS 代替 一抗作阴性对照, 最后在显微镜下 $(\times 10)$ 每张片随机 计数 5 个不重叠视野下阳性细胞数, 每组计数 6 张片 $(n=6)$. 细胞分组: (1) NRK-49F 细胞. 不加 TGF- $\beta 1$ 和加 $5 \mathrm{ng} / \mathrm{mL}$ TGF- $\beta 1$ 分别刺激 24 和 $48 \mathrm{~h}$; (2) $C T G F$ 基因转染细胞. 不加 TGF- $\beta 1$ 和加 $5 \mathrm{ng} / \mathrm{mL}$ TGF- $\beta 1$ 分别刺激 24 和 $48 \mathrm{~h}$.

( V ) Western blot 蛋白分析. 根据免疫组织化 学染色结果, 0 选择细胞分组: (1) NRK-49F 细胞. 不 
加 TGF- $\beta 1$ 和加 $5 \mathrm{ng} / \mathrm{mL}$ TGF- $\beta 1$ 刺激 $48 \mathrm{~h}$; (2) $C T G F$ 基因转染细胞. 不加 TGF- $\beta 1$ 和加 $5 \mathrm{ng} / \mathrm{mL}$ TGF- $\beta 1$ 刺激 $48 \mathrm{~h}$. 细胞裂解后, 取 $50 \mu \mathrm{g}$ 总蛋白以 $10 \%$ SDSPAGE 凝胶电泳分离, 电转移蛋白至 PVDF 膜上, $5 \%$ 无脂奶粉封闭, 与抗 $\alpha$-平滑肌肌动蛋白抗体孵育杂交, 最后以化学发光法(ECL, 英国 Amersham-Pharmacia 公 司)显示结果.

(vi) 逆转录-PCR. 按 Trizol 试剂 (美国 Gibco BRL 公司) 说明书方法提取细胞总 RNA, 并按逆转 录试剂盒(美国 Promega 公司)说明书方法先逆转录 合成第 1 条 cDNA, 再以此 cDNA 为模板进行 PCR 反应. PCR 产物经 $2 \%$ 琼脂糖凝胶电泳分离, 应用凝 胶成像系统 (electrophoresis documentation and analysis system 120, 美国 Kodak 公司)分析, 以目的 胶原 III 片段/内参照 GAPDH 片段的条带光密度的比值 作比较. 引物序列和扩增片段分别为: 胶原蛋白 III, F(正链): 5' -CGAGGTAACAGAGGTGAAAGA-3', $\mathrm{R}$ (负链): 5' -AACCCAGTATTCTCCGCTCTT-3'，349 bp; GAPDH, F: 5'-AATGCATCCTGCACCACCAA-3', R: 5'-GTAGCCATATTCATTGTCATA-3'，515 bp. 至 少重复 3 次独立的 RT-PCR 全过程.

(vii) 数据分析. 实验结果用平均值 \pm 标准差 $(\bar{x} \pm \mathrm{SD})$ 表示, 统计学处理采用 $t$ 检验.

\section{2 实验结果}

\section{1 基因转染成功并稳定表达 CTGF 的细胞系的鉴定}

转染 $C T G F$ 基因的细胞有 $\mathrm{CTGF}$ 蛋白表达; 转染 空载体质粒和正常 NRK-49F 细胞无 CTGF 蛋白表达 (图 1(a)), 说明稳定表达 CTGF 的细胞系建立成功.

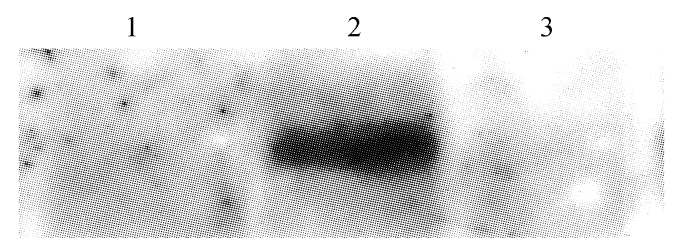

(a)

12

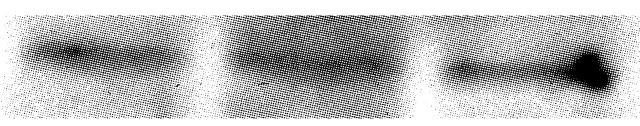

(b)

图 $1 \mathrm{CTGF}$ 蛋白在细胞表达的变化

(a) 基因转染细胞鉴定. 1 示空载体转染, 2 示 $C T G F$ 基因转染, 3 示正 常 NRK-49F 细胞. (b) 转染 $C T G F$ 基因的细胞在不同培养液时 CTGF 蛋白表达的变化. 1 示 $5 \% \mathrm{FBS}, 2$ 示 $5 \mathrm{mg} / \mathrm{mL}$ TGF- $\beta 1$ 刺激 $24 \mathrm{~h}, 3$ 示 5 $\mathrm{ng} / \mathrm{mL}$ TGF- $\beta 1$ 刺激 $4 \mathrm{~h}$ /engine.scichina.com/

同时 CTGF 稳定表达细胞在 5\% FBS DMEM 培养和 含 $5 \mathrm{ng} / \mathrm{mL}$ TGF- $\beta 1$ 的培养液中, CTGF蛋白表达量无 改变(图 1(b)).

\section{2 成肌纤维细胞数和 $\alpha$-平滑肌肌动蛋白表达}

正常 NRK-49F 细胞和转导空载体质粒的细胞不 论是否加 TGF- $\beta 1$ 刺激及刺激时间长短, 均未见 $\alpha$-平 滑肌肌动蛋白免疫染色阳性细胞. $C T G F$ 基因转染细 胞在 5\%FBS DMEM 培养时可见阳性细胞 $(1.53 \pm$ 0.23 (高倍视野)), 在 $5 \mathrm{ng} / \mathrm{mL}$ TGF- $\beta 1$ 刺激 $24 \mathrm{~h}$ 阳性 细胞增加 $(2.30 \pm 0.14$ (高倍视野)), 与正常培养时阳 性细胞数相比有显著差异 $(P<0.01)$; 刺激 $48 \mathrm{~h}$ 阳性 细胞显著增加 $(8.73 \pm 0.90$ (高倍视野)), 与 $5 \mathrm{ng} / \mathrm{mL}$ TGF- $\beta 1$ 刺激 $24 \mathrm{~h}$ 时阳性细胞数相比有显著差异 $(P<$ 0.001 , 图版 I, 附本刊后).

$C T G F$ 基因转染细胞在 5\%FBS DMEM 培养时即 有 $\alpha$-SMA 蛋白表达, 经 $5 \mathrm{ng} / \mathrm{mL}$ TGF- $\beta 1$ 刺激 $48 \mathrm{~h}$ 后表达明显增加; NRK-49F 细胞和转导空载体质粒 的细胞在 5\%FBS DMEM 和 $5 \mathrm{ng} / \mathrm{mL}$ TGF- $\beta 1$ 刺激 $48 \mathrm{~h}$ 均无 $\alpha$-SMA 蛋白表达(图 2).

1

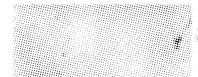

图 2 不同培养状况时细胞表达 $\alpha-S M A$ 蛋白水平比较 1 示空载体转染细胞 $5 \mathrm{ng} / \mathrm{mL}$ TGF- $\beta 1$ 刺激 $48 \mathrm{~h} ; 2$ 示 NRK- $49 \mathrm{~F}$ 细胞 $5 \mathrm{ng} / \mathrm{mL}$ TGF- $\beta 1$ 刺激 $48 \mathrm{~h}, 3$ 示 $C T G F$ 基因转染细胞 5\% FBS DMEM $48 \mathrm{~h}, 4$ 示 $C T G F$ 基因转染细胞 $5 \mathrm{ng} / \mathrm{mL}$ TGF- $\beta 1$ 刺激 $48 \mathrm{~h}$

\section{3 细胞表达间质胶原 III mRNA 水平}

NRK-49F 细胞在 5\% FBS 正常培养时表达胶原 III $\mathrm{mRNA}$, 经 $5 \mathrm{ng} / \mathrm{mL}$ TGF- $\beta 1$ 刺激 $24 \mathrm{~h}$ 后表达水平升 高; $C T G F$ 基因转染细胞的胶原 III mRNA 在经 $5 \mathrm{ng} / \mathrm{mL}$ TGF- $\beta 1$ 刺激 $24 \mathrm{~h}$ 后表达显著增加(图 3(a)). 空载体转染细胞和正常 NRK-49F 细胞的胶原 III mRNA 水平一致, 没有明显变化 (未显示). 胶原 III 片 段/GAPDH 片段比值见图 3(b).

\section{3 讨论}

Grotendorst 等人 ${ }^{[4]}$ 于 1991 年克隆了结缔组织生 长因子 (CTGF), 并发现它能刺激成纤维细胞增殖; 在 TGF- $\beta 1$ 刺激下成纤维细胞又能合成 CTGF，而上 皮细胞不具有此反应, 说明 CTGF 是调控成纤维细 胞生长的重要蛋臽 ${ }^{[5]}$. 教肾脏纤维化过程的早期, 可 


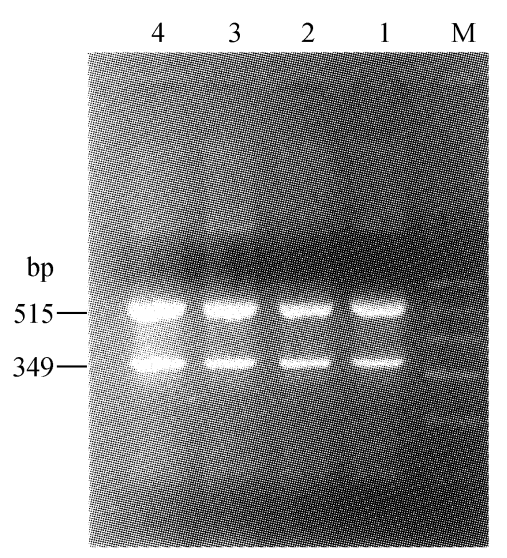

(a)

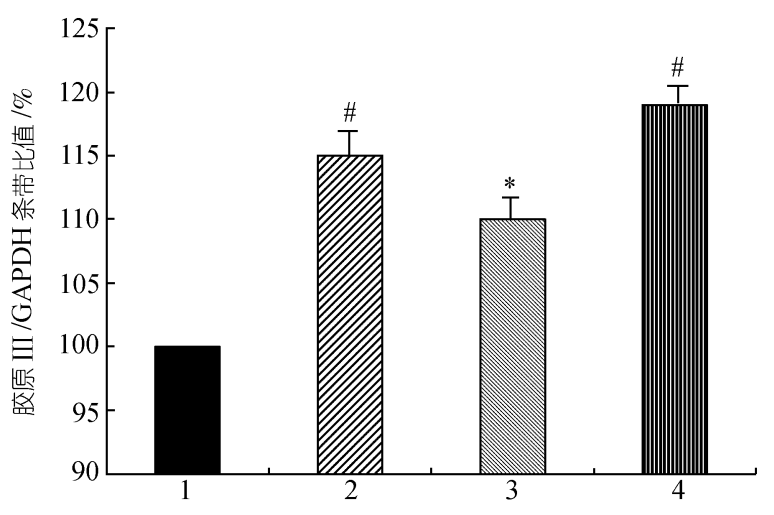

(b)

图 3 细胞表达胶原 III mRNA 水平变化

(a) 胶原 III mRNA 的 RT-PCR 电泳结果. (b) 胶原 III/GAPDH 条带的 光密度比值. M 示 DNA 分子量标准 $(600,500,400,300,200$ bp), 1 示 对照即培养 $24 \mathrm{~h}$ 的 NRK- $49 \mathrm{~F}$ 细胞, 2 示含 $5 \mathrm{ng} / \mathrm{mL}$ 的 TGF- $\beta 1$ 刺激 $24 \mathrm{~h}$ 的 NRK-49F 细胞, 3 示培养 $24 \mathrm{~h}$ 的 CTGF 转染细胞, 4 示含 $5 \mathrm{ng} / \mathrm{mL}$ 的 TGF- $\beta 1$ 刺激 $24 \mathrm{~h}$ 的 CTGF 转染细胞. \#示 $P<0.01$, *示 $P<0.05$, 与 对照比较

以观察到肾间质区存在一种表达骨架蛋白一 $\alpha$-平 滑肌肌动蛋白的细胞, 称为成肌纤维细胞. 它是介 于成纤维细胞和平滑肌细胞之间的终末分化细胞, 这类细胞是肾间质细胞外基质的主要来源，其数量 的多少与间质纤维化轻重程度密切相关 ${ }^{[6,7]}$, 可以作 为判断长期预后的一个很好的指标 ${ }^{[2,6]}$. 正常肾脏几 乎没有成肌纤维细胞, 疾病状态下这种细胞的来源 还不能肯定, 有研究表明 ${ }^{[8]}$ 多种细胞生长因子如 TGF- $\beta$, PDGF, IL- 1 能刺激肾小球系膜细胞、肾小管 上皮细胞发生表型转化，但迄今没有在体外细胞实 验上直接显示肾间质成纤维细胞能被活化转为成肌 纤维细胞. 尽管 Ito 等人 ${ }^{[3]}$ 在不同肾病患者肾穿刺标 本中, 发现表达 CTGF mRNA 的细胞均呈 $\alpha$-平滑肌
肌动蛋白免疫染色阳性, 但 CTGF 对成肌纤维细胞 的形成有何影响尚不清楚. 我们成功地把 $C T G F$ 基 因转人肾间质成纤维细胞, 得到稳定高表达 CTGF 蛋白的细胞株, 并直接观察 $C T G F$ 基因对此细胞的 生物效应. 发现 CTGF 能够诱导成纤维细胞转化为 成肌纤维细胞, 在有 TGF- $\beta 1$ 存在时, 成肌纤维细胞 数明显增多, 其表面标记蛋白 $\alpha$-平滑肌肌动蛋白水 平亦显著增加, 这是首次发现 CTGF 具有这样的生 物学效应. 因为正常 NRK-49F 细胞和空载体转导细 胞在 TGF- $\beta 1$ 刺激后没有观察到成肌纤维细胞, 也未 检测到 $\alpha$-平滑肌肌动蛋白表达, 因此可以排除 TGF- $\beta 1$ 对细胞的直接刺激作用. 转染 CTGF 的细胞 是过表达(over-express) CTGF 蛋白, 所以 CTGF 稳定 表达细胞内 CTGF 蛋白水平不受 TGF- $\beta 1$ 刺激的影 响; TGF- $\beta 1$ 不能直接诱导成纤维细胞表型转化, 却 能增强 CTGF 诱导肌成纤维细胞生成的效应, 更进 一步证实了 $\mathrm{CTGF}$ 是 TGF- $\beta 1$ 的下游作用蛋白因 ${ }^{[9,10]}$, 但是 CTGF 和 TGF- $\beta 1$ 之间的这种调控机制 尚不清楚. 本研究也观察到 CTGF 诱导细胞表型转 化后细胞内胶原 III mRNA 水平增加, 说明成肌纤维 细胞增加后, 细胞外基质合成也增多, 进一步从细 胞水平证实了以往在肾病患者的肾组织 ${ }^{[3]}$ 和 $5 / 6$ 肾切 除肾病、糖尿病肾病动物模型 ${ }^{[11,12]}$ 上的观察发现, 即 在慢性肾纤维化晚期，肾间质内 CTGF mRNA 水平 高低与间质细胞外基质沉积程度呈正相关的现象. 因此，肾间质损伤后肾细胞和浸润的炎症细胞产生 细胞因子 TGF- $\beta 1$, 作用于成纤维细胞使之合成分泌 CTGF, CTGF 既能介导 TGF- $\beta$ 的促纤维化效应, 又能 诱发成肌纤维细胞的生成, 从而在肾间质纤维化慢 性进展中起重要的作用.

TGF- $\beta$ 是组织纤维化病变中最重要的促发因子 之一已经得到公认，曾有许多研究显示阻断 TGF- $\beta$ 的表达或活性, 可以减轻组织纤维化; 但是由于 TGF- $\beta$ 对多种细胞类型具有广泛的生物学活性, 如 抑制细胞生长、免疫抑制、细胞凋亡等, 因此在获得 抗组织纤维化效应时, 也不可避免地带来诸如细胞 生长失控、免疫失调、严重炎症甚至死亡等副反应 ${ }^{[13]}$, 限制了其在防治肾脏纤维化中的应用. 我们的研究 结果阐明了 CTGF 可以作为防治肾纤维化的新的靶 位蛋白, 它具有独特的促肾间质成纤维细胞增殖、细 胞外基质增加和成纤维细胞表型转化, 即促肾间质 纤维化效应, 所以采取抑制或干预 CTGF 生物学效 应的药物或措施, 不会产生像干预 TGF- $\beta$ 后存在的 副反应，这为临床上找到安全、有效延缓肾脏纤维化 途径提供了新的思路. 
致谢 本实验得到屈否技术员的大力帮助，作者对此深表 感谢. 本工作为北京大学 “985”行动计划、教育部留学回 国人员科学研究启动基金和北京大学第一医院留学归国 科学研究启动基金资助项目.

\section{参考文献}

1 Eddy A A. Molecular basis of renal fibrosis. Pediatri Nephrol, 2000, 15: 290 302

2 Badid C, Vincent M, Fouque D, et al. Myofibroblast: a prognostic marker and target cell in progressive renal disease. Renal Fail, 2001, 23: 543 549

3 Ito Y, Aten J, Bende R J, et al. Expression of connective tissue growth factor in human renal fibrosis. Kidney Int, 1998, 53: $853 \sim 861$

4 Bradham D M, Igarashi A, Grotendorst G R. Connective tissue growth factor: a cystein-rich mitogen secreted by human vascular endothelial cells is related to the SRC-induced immediate early gene product CEF-10. J Cell Biol, 1991, 114: 1285 1294

5 Goldschmeding R, Aten J, Ito Y, et al. Connective tissue growth factor: just another factor in renal fibrosis? Nephrol Dial Transplant, 2000, 15: 296 299

6 Boukhalfa G, Desmouliere A, Rondeau E, et al. Relationship between alpha-smooth muscle actin expression and fibrosis changes in human kidney. Exp Nephrol, 1996, 4: 241 247
7 王玉，李晓玫，邹万忠，等.人类肾小球肾炎中肾小管及间质 细胞表型转化的研究. 中华肾脏病杂志, 2000, 16: 7 10

8 Gupta S, Clarkson M R, Duggan J, et al. Connective tissue growth factor: potential role in glomerulosclerosis and tubulointerstitial fibrosis. Kidney Int, 2000, 58: 1389 1399

9 Blom I E, van Dijk A J, Wieten L, et al. In vitro evidence for differential involvement of CTGF, TGF $\beta$, and PDGF-BB in mesangial response to injury. Nephrol Dial Transplant, 2001, 16: $1139 \sim 1148$

10 Grotendorst G R. Connective tissue growth factor: a mediator of TGF-beta action on fibroblasts. Cytokine Growth Factor Rev, 1997, 8: 171 179

11 Gore E, Foster S, Hazen-Martin D, et al. Immunohistochemical analysis of CTGF, TGF $\beta$ \& TGF $\beta$ receptor in the remnant kidney model of chronic renal failure. J Am Soc Nephrol (Abs), 2000, 11: 528

12 Wang S N, Denichilo M, Brubaker C, et al. Connective tissue growth factor in tubulointerstitial injury of diabetic nephropathy. Kidney Int, 2001, 60: 96 105

13 Gorelik L, Flarell R A. Abrogation of TGF $\beta$ signaling in T cells leads to spontaneous $\mathrm{T}$ cells differentiation and autoimmunal disease. Immunity, 2000, 12: 171 181

(2001-09-05 收稿, 2001-11-23 收修改稿) 


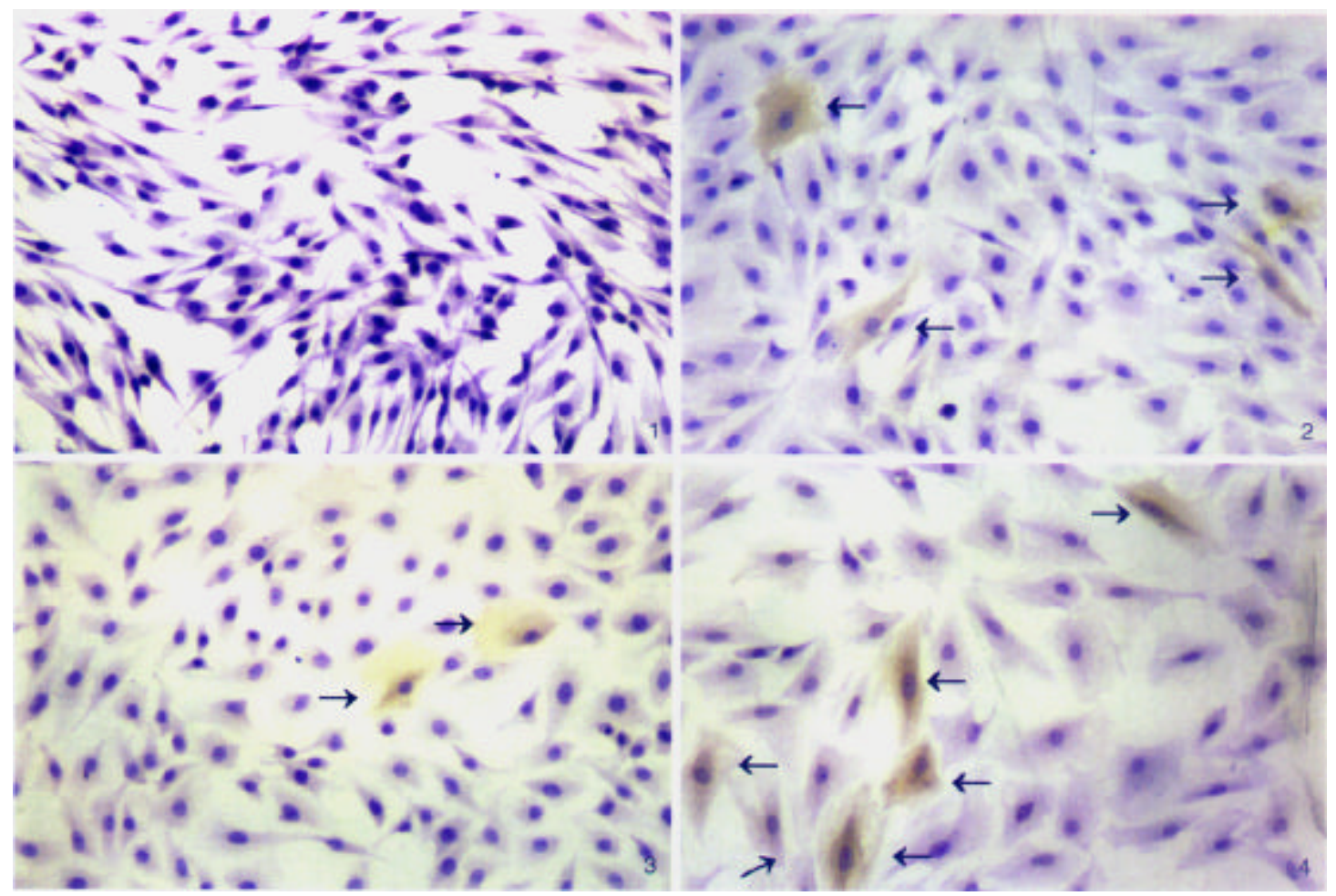

\footnotetext{
$\alpha$-平滑肌肌动蛋白免疫组织化学染色( $\mathrm{ABC}$ 法)
}

1 示 NRK-49F 细胞以 $5 \mathrm{ng} / \mathrm{mL}$ TGF- $\beta 1$ 刺激 $48 \mathrm{~h} ; 2$ 示 $C T G F$ 基因转染细胞在 $5 \%$ FBS DMEM 培养 $24 \mathrm{~h} ; 3$ 示 $C T G F$ 基因转染细胞以 $5 \mathrm{ng} / \mathrm{mL}$ TGF- $\beta 1$ 刺激 $24 \mathrm{~h} ; 4$ 示 $C T G F$ 基因转染细胞以 $5 \mathrm{ng} / \mathrm{mL}$ TGF- $\beta 1$ 刺激 $48 \mathrm{~h}$. 箭头示胞浆棕黄色染色为阳性细胞, 苏木素复染细胞核, $\times 10$ 